\title{
First Principles Calculations of Hydrogen Storage on Calcium-Decorated, Boron-Doped Bilayer Graphene
}

\author{
Xiaojing Zhu \\ Shanghai University of Engineering Science, Shanghai, China \\ Email:m17621329279@qq.com
}

How to cite this paper: Zhu, X.J. (2018) First Principles Calculations of Hydrogen Storage on Calcium-Decorated, Boron-Doped Bilayer Graphene. Journal of Materials Science and Chemical Engineering, 6, 1-12. https://doi.org/10.4236/msce.2018.611001

Received: January 18, 2018

Accepted: November 27, 2018

Published: November 30, 2018

Copyright $\odot 2018$ by author and Scientific Research Publishing Inc. This work is licensed under the Creative Commons Attribution International License (CC BY 4.0).

http://creativecommons.org/licenses/by/4.0/

\begin{abstract}
In this paper, the adsorption and storage of hydrogen on calcium-decorated, boron-doped bilayer graphene was investigated using first principles calculation. The calcium-decorated bilayer graphene was investigated and it was shown that the binding energy of $\mathrm{H}_{2}$ molecule adsorbed on the calcium-decorated bilayer graphene is $-0.02 \mathrm{eV}$ and the energy does not belong to reversible usage range of $-0.2--0.6 \mathrm{eV}$. Substitutional boron doping can improve the adsorption energy of Ca to bilayer graphene with the empty $p_{z}$ orbital of boron atoms. Our calculations show that calcium atoms can be solidly adsorbed on the interlayer $(\mathrm{Ca} / \mathrm{B} / \mathrm{Graphene})$ and outerlayer $(2 \mathrm{Ca} / \mathrm{B} / \mathrm{Graphene}$ and $3 \mathrm{Ca} / \mathrm{B} / \mathrm{Graphene}$ ) of $\mathrm{B}$-doped bilayer graphene. Hydrogen molecule binds with $\mathrm{Ca} / \mathrm{B} / \mathrm{Graphene}$, $2 \mathrm{Ca} / \mathrm{B} / \mathrm{Graphene}$ and $3 \mathrm{Ca} / \mathrm{B} / \mathrm{Graphene}$ system with an energy that belongs to reversible usage range of $-0.2--0.6 \mathrm{eV}$. The overlap between $\mathrm{Ca} 3 \mathrm{~d}$ and $\mathrm{H}_{2} \sigma$ orbitals just below the Fermi energy demonstrates the charge transfer between the $\mathrm{Ca}$ atom and the $\mathrm{H}$ atom and the role of hybridization of the $3 \mathrm{~d}$ orbita of $\mathrm{Ca}$ with the $\sigma$ orbitals of $\mathrm{H}_{2}$ in efficient adsorption of hydrogen molecules. The charge from hydrogen bonding orbital transfers to empty $3 \mathrm{~d}$ orbitals of the $\mathrm{Ca}$ atom, and then from the $3 \mathrm{~d}$ orbitals of the $\mathrm{Ca}$ atom donated to $\mathrm{H} 2 \sigma^{*}$ antibonding orbital. Hydrogen moleculars can be adsorbed on the interlayer and outerlayer of Ca-decorated B-doped bilayer graphene.
\end{abstract}

\section{Keywords}

Hydrogen Storage, Calcium-Decorated, Boron-Doped, Bilayer Graphene, Interlayer, Outerlayer 


\section{Introduction}

Energy crisis and environmental problems are the focus of the world, and also the research focus of researchers. As a kind of clean energy, hydrogen can be regarded as the most ideal secondary energy, because of its abundant reserves, pollution-free, easily synthesized, renewability, sustainability and high utilization rate [1] [2] [3] [4] [5]. However, hydrogen energy is mainly in the gaseous form of hydrogen and it is very easy to explode. Therefore, the storage and transportation of hydrogen energy has become a hot topic in academic research in various countries. Hydrogen can be easily absorbed and released at room temperature when the optimal hydrogen adsorption energy is between -0.2 $-0.6 \mathrm{eV} / \mathrm{H}_{2}$ [6] [7], and hydrogen can be stored efficiently and reversibly. The range of energy is intermediate between physical adsorption and chemical adsorption. However, the adsorption energy of pure graphene and hydrogen is between $-0.01--0.09 \mathrm{eV} / \mathrm{H}_{2}$, which cannot meet the requirement of efficient reversible storage of hydrogen. There have been a lot of researches on hydrogen storage materials such as metal hydride, carbon material and metal organic frame. However, these technologies have some disadvantages, such as low capacity, irreversible or security problems. In recent years, carbon nanomaterials with high specific surface area have been widely studied in hydrogen storage.

Graphene is a two-dimensional carbon sheet that is connected by the hybridization of $\mathrm{sp}^{2}$, arranged into a hexagonal honeycomb lattice. The thickness of graphene is about $0.335 \mathrm{~nm}$, which is the thinnest of two-dimensional nanomaterials [8] [9]. Since the discovery of graphene, it has received a lot of attention from fundamental nanoscience and applicable nanotechnology [10]. Due to the successful production of single-layer graphene, through experiment and theoretical study, many efforts have been devoted to the study of graphene's two-dimensional materials [11] [12] [13]. Because of its stable structure, light weight, high specific surface area, easy modification and unique physical and chemical properties, graphene has become a potential hydrogen storage medium. The target value of the hydrogen storage system as specified by the department of energy (DOE) includes a weight capacity of $10 \mathrm{wt} \%$ and volume 4.0 vol\% by 2017. In recent years, many studies have focused on functionalizing the surface of carbon nanostructures with transition metals (TMs) to enhance binding energy and hydrogen storage capacity of carbon nanostructures. However, because the cohesive energy of the TM atom itself is greater than the binding energy on the surface of carbon nanomaterials, a cluster of TM atoms can change the properties of hydrogen bonds, and the hydrogen storage capacity is greatly reduced. For estimation of capacity, TM atoms were assumed to be evenly distributed on the surface.

Calcium is the first atom to have an empty $3 \mathrm{~d}$ orbit and has recently become the preferred material for improving the storage capacity of carbon nanostructures [14] [15] [16] [17]. Recently, it has been found that the Ca and other alkali metal atoms can adsorb on the pure carbon nanostructure without clustering 
and have high hydrogen storage capacity [18]. The empty $p_{z}$ orbital of boron is used as a strong charge receptor, thus improving the adsorption capacity of $\mathrm{Ca}$ on graphene. Boron can be easily combined in the hexagonal structure of graphene, and boron doped graphene has been synthesized experimentally. Previous researchers have done a great deal of research on the adsorption of hydrogen on calcium-decorated graphene, but little research has been done on bilayer graphene. In this paper, we mainly study the adsorption of hydrogen on calcium-decorated, boron-doped bilayer graphene. We simulated and analyzed the structure, hydrogen storage performance and state density of the modified bilayer graphene, and found the stable configuration and optimal hydrogen storage performance.

\section{Calculation Details}

Our calculations were carried out using DFT and VASP software packages. In the study, the supercell a $3 \times 3$ bilayer graphene contains 36 carbon atoms. The interaction potential of ions and electrons is approximated by using the projection augmented wave method. The exchange-correlation functional is approximated with the local density approximation (LDA). According to the monkhorst-pack plan, the Brillouin zone was sampled by $(5 \times 5 \times 1)$ special mesh points in $\mathrm{K}$ space for $(3 \times 3)$ bilayer graphene cells [19]. Considering the computational cost, the energy cutoff value of the plane wave is chosen to be $450 \mathrm{eV}$. For all the geometry optimization using conjugate gradient (CG) algorithm, convergence criterion was that we allow all internal coordinates relaxed until the most residual force was less than $0.01 \mathrm{eV} / \AA$ and total pressure of the system was less than $0.1 \mathrm{kbar}$ per unit cell. The convergence criterion of energy is selected to be less than $10^{-7} \mathrm{eV}$. The theoretical estimate of pure graphene lattice constants is $2.46 \AA$. In order to avoid the interaction between the adjacent layers of graphene, the height of vacuum layer in the $\mathrm{z}$ direction of the graphene sheet is 15 Å.

Bilayer graphene has two configurations: $\mathrm{AA}$ and $\mathrm{AB}$ stackings. We construct the structures of bilayer graphene with both $\mathrm{AB}$ and $\mathrm{AA}$ stackings as shown in Figure 1. AA-type bilayer graphene is refers to the structure of the upper layer and lower layer are consistent, AB-type bilayer graphene is a carbon atom of the

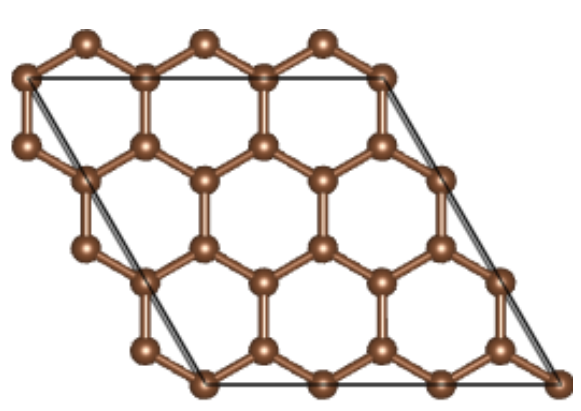

AA stacking

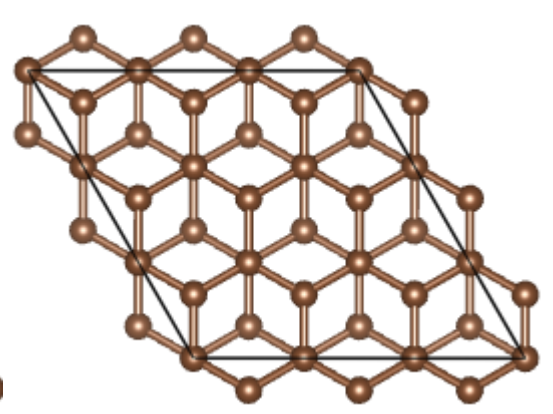

AB stacking

Figure 1. Typical configurations of bilayer graphene. 
upper layer in the center of hexagonal lattice of the lower layer.

The binding energies of $\mathrm{Ca}$ atom adsorbed on the bilayer graphene structures are calculated as following equation

$$
E_{\text {ad-Ca }}=E_{\mathrm{Ca} / \mathrm{C}}-E_{\mathrm{Ca}}-E_{\mathrm{C}}
$$

The binding energies of Ca-decorated B-doped bilayer graphene structures are calculated as following equation

$$
E_{\mathrm{ad}-\mathrm{Ca}}=E_{\mathrm{Ca} / \mathrm{B} / \mathrm{C}}-E_{\mathrm{Ca}}-E_{\mathrm{B} / \mathrm{C}}
$$

where $E_{\mathrm{Ca} / \mathrm{C}}$ is the total energy of the system with $\mathrm{Ca}$ atom adsorbed on the bilayer graphene, $E_{\mathrm{Ca} / \mathrm{B} / \mathrm{C}}$ is the total energy of Ca-decorated B-doped bilayer graphene system, $E_{\mathrm{B} / \mathrm{C}}$ is the total energy of the system with $\mathrm{B}$ atom adsorbed on the bilayer graphene, $E_{\mathrm{C}}$ is the total energy of bilayer graphene sheet, and $E_{\mathrm{Ca}}$ is the total energy of an isolated $\mathrm{Ca}$ atom.

And the average adsorption energy of $\mathrm{H}_{2}$ molecules on Ca-decorated B-doped bilayer graphene system is defined as

$$
E_{\text {ad- } \mathrm{H}_{2}}=\frac{E_{\mathrm{Ca} / \mathrm{B} / \mathrm{G} / n \mathrm{H}_{2}}-E_{\mathrm{Ca} / \mathrm{B} / \mathrm{G}}-n \mathrm{H}_{2}}{n}
$$

where $E_{\mathrm{Ca} / \mathrm{B} / \mathrm{G} / n \mathrm{H}_{2}}, \quad E_{\mathrm{Ca} / \mathrm{B} / \mathrm{G}}$ and $E_{\mathrm{H}_{2}}$ denote the total energy of the Ca-decorated B-doped bilayer graphene system with $\mathrm{n}_{2}$ molecules adsorbed, the total energy of the system with $(n-1) \mathrm{H}_{2}$ molecules adsorbed, the total energy of Ca-decorated B-doped bilayer graphene system and the total energy of free $\mathrm{H}_{2}$ molecule, respectively.

\section{Results and Discussion}

\subsection{Stable Configuration of Ca Adsorption}

We first studied the atomic structure pure AA-type and AB-type bilayer gaphenes. The optimized interlayer distance of AA-type bilayer gaphenesis $3.55 \AA$, The optimized interlayer distance of AB-type bilayer gaphenesis $3.33 \AA$, which is consistent with other results [20] [21]. The bond length between two carbon atoms is $1.41 \AA$ for $\mathrm{AA}$ and $\mathrm{AB}$ structure. In the paper, we study atomic structure of AA stacking bilayer graphene. According to the calculations in the previous section, the interlayer distance of bilayer graphene is too small to store hydrogen. So we need to modify the bilayer graphene.

Calcium is the first atom to have an empty $3 \mathrm{~d}$ orbit and has recently become the preferred material for improving the storage capacity of carbon nanostructures.In this paper, a $\mathrm{Ca}$ atom is decorated in the middle of two layers of graphene, and the adsorption of $\mathrm{Ca}$ atoms can be calculated by using Equation (1). The binding energies of $\mathrm{Ca}$ atom adsorbed on bilayer graphene structures can be $2.48 \mathrm{eV}$, which is greater than the internal cohesive energy $(1.8 \mathrm{eV})$, indicating that clusters are not easily formed. But the total energy of the Ca-decorated bilayer graphene system with one $\mathrm{H}_{2}$ molecule adsorbed is $-0.02 \mathrm{eV}$, and this value is not between $-0.2 \mathrm{eV}--0.6 \mathrm{eV}$. So the $\mathrm{Ca}$-decorated bilayer graphene system can't store hydrogen. Because the empty $p_{z}$ orbital of boron is a strong electron 
acceptor, it can improve the adsorption energy of Ca to bilayer graphene. Boron can easily be substituted in graphene's hexagonal structure, while B-doped graphene is synthesized experimentally. Considering the periodicity, B is substituted for the same position of the upper graphene and the lower graphene. The total energy of the Ca-decorated B-doped bilayer graphene system was $-5.72 \mathrm{eV}$ by Equation (2). We found that the adsorption energy of B-doped system was smaller and more stable. The bond lengths between $B$ and $C$ atoms in B-doped bilayer graphene are found to be all $1.47 \AA$ for AA stackings. The interlayer distances of B-doped bilayer graphene are found to be changed from $3.55 \AA$ to 3.30 $\AA$, compared with those of pristine graphene. On the other hand, the $\mathrm{B}-\mathrm{C}$ bond length is much longer than the $\mathrm{C}-\mathrm{C}$ bond length in a pristine bilayer graphene, and it is almost the same value as that of the B-doped monolayer grapheme [22] [23]. As we have shown in Figure 2, pure graphene showed typical semiconductor band structure properties, the B-doped graphene of the density of states are shifted above the Fermi level, as the empty $p_{z}$ orbital of the boron acts as a strong charge accepting center. As a result, boron-doped graphene forms an electron-deficient structure and it can easily accept excess electrons. Graphene is converted from a semiconductor to a metal property.

As show in main information Figure 3 and Figure 4, we used $\mathrm{Ca}$ atom to modify B-doped bilayer graphene, considering the relative positions of $\mathrm{Ca}$ atom and $\mathrm{B}$ atom, there are five different adsorption sites. In order to increase the calculation speed, spin-polarization has not been considered first in determining the stable position of a $\mathrm{Ca}$ atom on B-doped graphene. The binding energy and interlayer distance of different configurations of the $\mathrm{Ca}$ atom on $\mathrm{B}$-doped bilayer graphene are listed in main information Table 1 . It is found that the most favorable adsorption site of a Ca atom on the bilayer graphene is the hollow site by

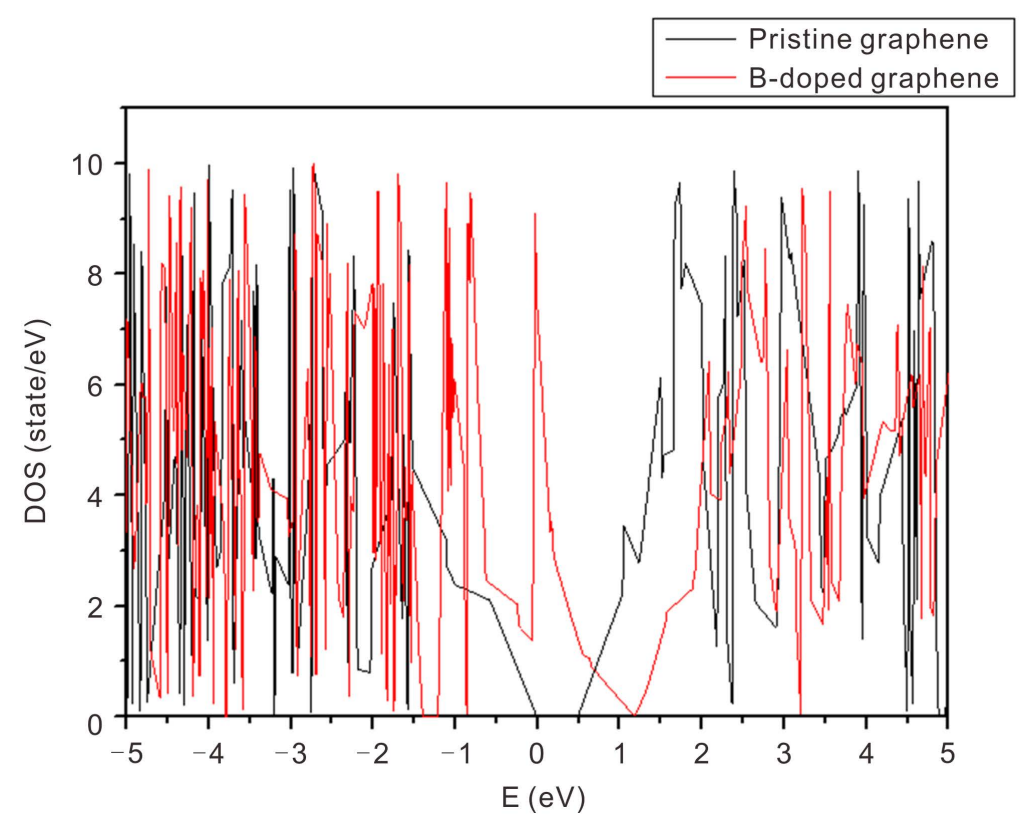

Figure 2. The density of states (DOS) for Pristine graphene and B-doped graphene. 


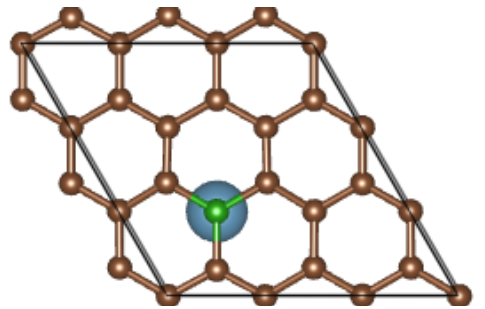

(a)

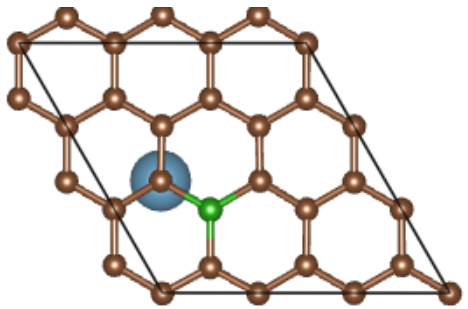

(b)

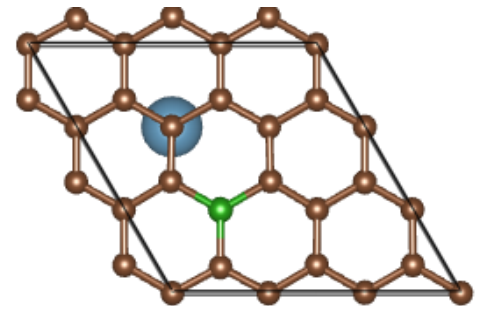

(c)

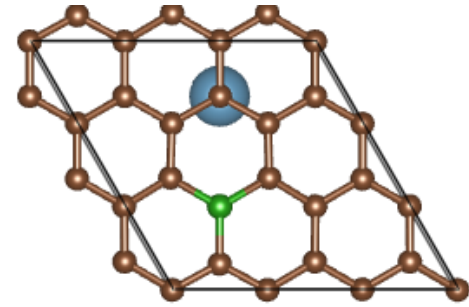

(d)

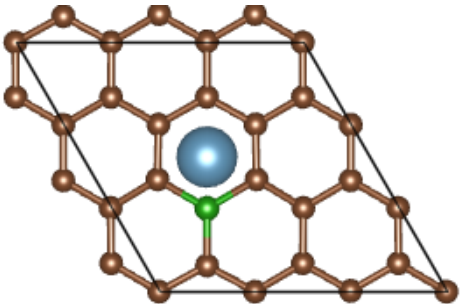

(e)

Figure 3. The top view of five different structures of calcium-decorated bilayer graphene (The grey represents $\mathrm{C}$, green for $\mathrm{B}$, and blue for $\mathrm{Ca}$ ).

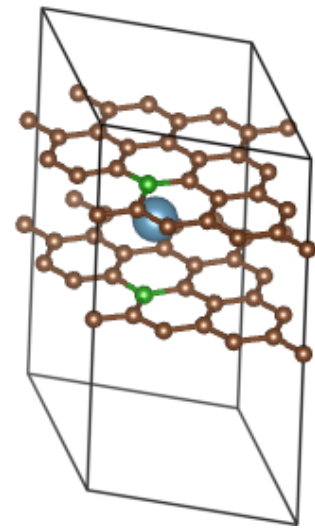

Figure 4. The side view of five different structures of calcium-decorated bilayer graphene (The grey represents $\mathrm{C}$, green for $\mathrm{B}$, and blue for $\mathrm{Ca}$ ).

Table 1. Binding energy and interlayer distance of different structures.

\begin{tabular}{ccc}
\hline & $\mathrm{E}_{\mathrm{b}} / \mathrm{eV}$ & $\mathrm{dc}-\mathrm{c} / \AA$ \\
\hline $\mathrm{a}$ & -5.35871 & 4.44489 \\
$\mathrm{~b}$ & -5.36365 & 4.36278 \\
$\mathrm{c}$ & -5.71103 & 4.41173 \\
$\mathrm{~d}$ & -5.71256 & 4.31396 \\
$\mathrm{e}$ & -5.71244 & 4.41117
\end{tabular}

calculation. At this time, the adsorption energy is minimum and the interlayer spacing is $4.4 \AA$. The Ca atoms on different initial position automatically move to the hollow site above the center of the hexagon after relaxation, and binding to the hollow site is energetically more favorable by analyzing the binding energies, and little deformation of bilayer graphene geometry structure can be seen. 


\section{2. $\mathrm{H}_{2}$ Molecule Adsorbed on Ca-Decorated B-Doped Bilayer Graphene System}

In order to understand the hydrogen storage capacity of the bilayer graphene, we calculated and analyzed the adsorption structure of a hydrogen molecule in this active position. The stable configuration of a hydrogen molecule between two layers of bilayer graphene is shown in Figure 5.

According to Figure 5, the optimal position of hydrogen adsorption on Ca-decorated B-doped bilayer graphene system is the hollow site, which is consistent with the experiment of ambo et al. [5]. In order to improve the hydrogen storage capacity of the bilayer graphene, we considered the modification of the interlayer and outerlayer of the bilayer graphene at the same time. Similar to the interlayer modification of Ca-decorated bilayer graphene, we assume that $\mathrm{Ca}$ atoms can be uniformly covered the bilayer graphene, and then study the adsorption behavior of $\mathrm{Ca}$ atoms in the bilayer graphene. Previous study found that the most stable position of the Ca atoms adsorbed on the bilayer Graphene is the hollow site, so it is decided to adsorb the Ca atom at the hollow position outside the double graphene. The stable configurations has been shown in Figure 6 . Based on the below construction of the optimized $2 \mathrm{Ca} / \mathrm{B} / \mathrm{Graphene}$ and $3 \mathrm{Ca} / \mathrm{B} / \mathrm{Graphene}$, we continued to study the adsorption capacity of the active system on hydrogen, and we simulated the adsorption of hydrogen molecules on this structure. Figure 7 is the stable configuration of bilayer graphene adsorbing hydrogen molecules.

According to Table 2, the continuous adsorption energies of $1 \mathrm{Ca} / \mathrm{B} / \mathrm{Graphene}$ $2 \mathrm{Ca} / \mathrm{B} / \mathrm{Graphene}$ and $3 \mathrm{Ca} / \mathrm{B} / \mathrm{Graphene}$ were found to be $-0.21 \mathrm{eV},-0.26 \mathrm{eV}$ and $-0.25 \mathrm{eV}$, respectively. The ideal adsorption energy of the hydrogen is -0.2 $\mathrm{eV} / \mathrm{H}_{2}$ to $-0.6 \mathrm{eV} / \mathrm{H}_{2}$, and the average adsorption energy of hydrogen of the three system is in this range, so it can control the behavior of hydrogen absorption and release under ambient temperature and pressure conditions. The range of energy is between physical adsorption and chemical adsorption. Compared with

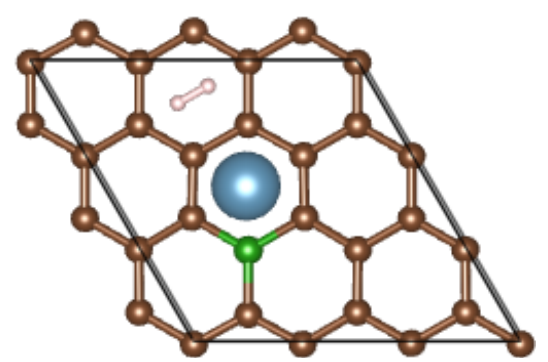

(a)

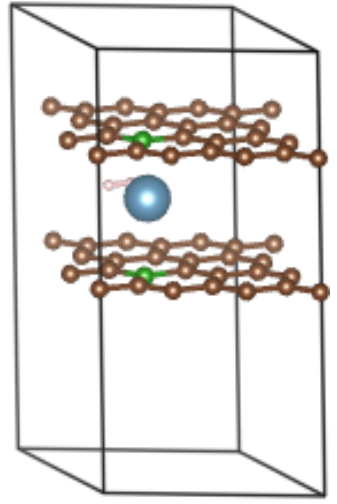

(b)

Figure 5. (a) (b) show a top view and the side view of the bilayer graphene by adsorbing a hydrogen molecule, respectively (violet represents a hydrogen atom, gray represents a carbon atom, blue represents a calcium atom, green represents a boron atom). 


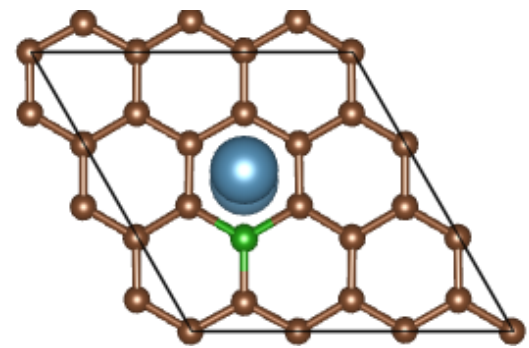

(a)

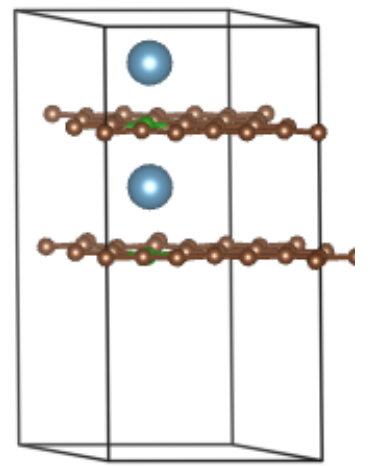

(c)

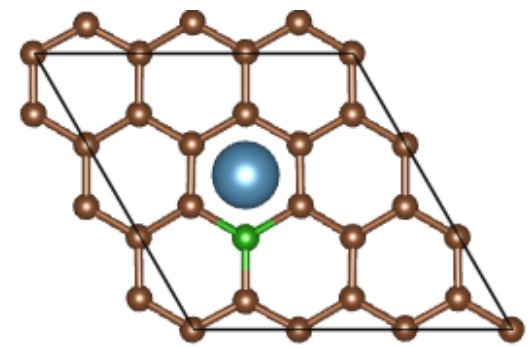

(b)

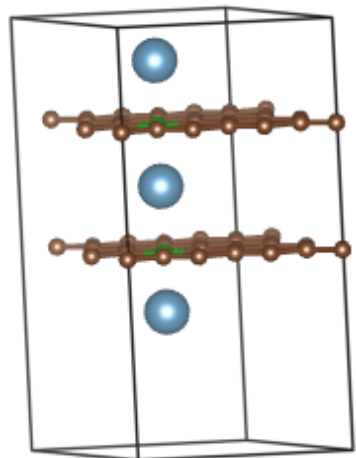

(d)

Figure 6. The figure represents the top and side views of the stable configuration of the $\mathrm{Ca}$ atoms on the bilayer graphene, respectively (gray represents a carbon atom, blue represents a calcium atom, green represents a boron atom).

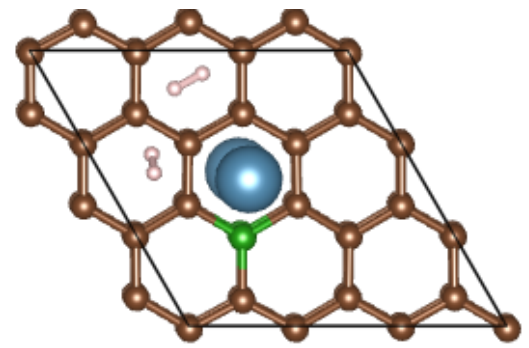

(a)

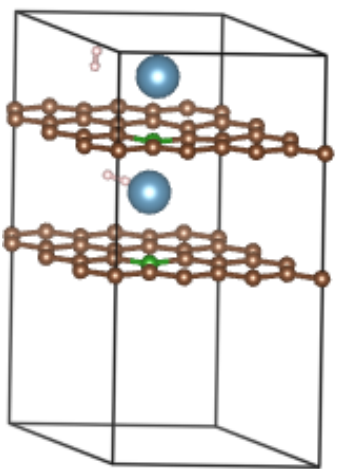

(c)

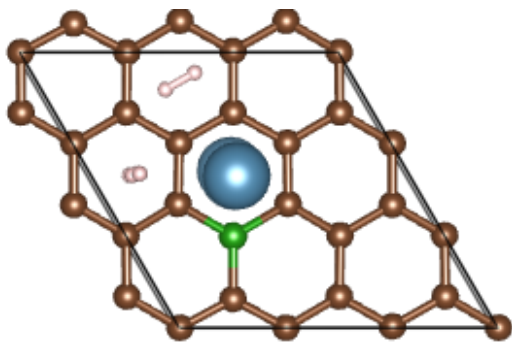

(b)

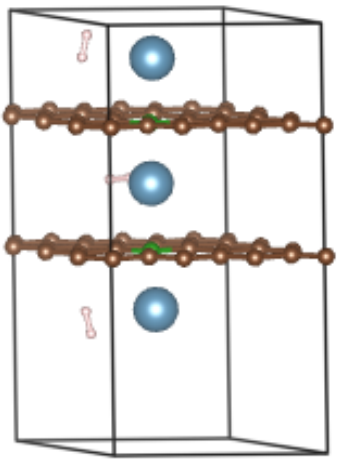

(d)

Figure 7. (a)-(d) show a top view and the side view of the bilayer graphene by adsorbing hydrogen molecules, respectively (violet represents a hydrogen atom, gray represents a carbon atom, blue represents a calcium atom, green represents a boron atom). 
Table 2. Average adsorption energy of hydrogen molecules, bond length of hydrogen molecule, distance between $\mathrm{Ca}$ atoms and graphene atoms and the interlayer distance of bilaye graphene.

\begin{tabular}{ccccc}
\hline & Ead- $\mathrm{H}_{2}(\mathrm{eV})$ & $\mathrm{dH}-\mathrm{H}(\AA)$ & $\mathrm{dCa}-\mathrm{G}(\AA)$ & $\mathrm{dC}-\mathrm{C}(\AA)$ \\
\hline $\mathrm{H}_{2} / 1 \mathrm{Ca} / \mathrm{B} / \mathrm{G}$ & -0.20891 & 0.7811 & 2.2476 & 4.4952 \\
$2 \mathrm{H}_{2} / 2 \mathrm{Ca} / \mathrm{B} / \mathrm{G}$ & -0.26127 & 0.78705 & 2.1495 & 4.4699 \\
$3 \mathrm{H}_{2} / 3 \mathrm{Ca} / \mathrm{B} / \mathrm{G}$ & -0.25889 & 0.78564 & 2.1955 & 4.4988 \\
\hline
\end{tabular}

isolated molecular hydrogen, the bond length of the system extends from $0.76 \AA$ to $0.78 \AA$.

The binding mechanism of $\mathrm{H}_{2}$ on Ca-decorated B-doped graphene has similarities to that of TM coated graphene. In order to further analyze the mechanism of action, we did the total and partial density of states of $1 \mathrm{H}_{2} / \mathrm{Ca} / \mathrm{B} / \mathrm{Graphene}$, $2 \mathrm{H}_{2} / 2 \mathrm{Ca} / \mathrm{B} / \mathrm{Graphene}$ and $3 \mathrm{H}_{2} / 3 \mathrm{Ca} / \mathrm{B} / \mathrm{Graphene}$ system, as shown in Figure 8. Figure 8 illustrates the partial density of states for the $\mathrm{H}_{2} \sigma$ and $\mathrm{Ca} 3 \mathrm{~d}$ orbitals for three cases, respectively. The overlap between $\mathrm{Ca} 3 \mathrm{~d}$ and $\mathrm{H}_{2} \sigma$ orbitals just below the Fermi energy demonstrates the charge transfer between the $\mathrm{Ca}$ atom and the $\mathrm{H}$ atom and the role of hybridization of the Ca $3 \mathrm{~d}$ orbital with the $\sigma$ orbitals of $\mathrm{H}_{2}$ in efficient adsorption of hydrogen molecules. First charge from hydrogen bonding orbital transfer to empty $3 \mathrm{~d}$ orbitals of the $\mathrm{Ca}$ atom, and then from the $3 \mathrm{~d}$ orbitals of the $\mathrm{Ca}$ atom donated to $\mathrm{H}_{2} \sigma^{*}$ antibonding orbital. Both can interact through charge transfer, this is a typical Kubas interaction. This mechanism will result in changes of electron cloud density of hydrogen molecules, including the decrease in bonding orbital and the increase in antibonding orbital. It will weaken the interaction of hydrogen atoms, which can lead to molecular hydrogen bond length increases, but not enough to decomposition hydrogen molecules. In this study, the hydrogen storage capacity can be greatly improved by modifying the interlayer and outerlayer of the bilayer graphene simultaneously.

\section{Conclusion}

In conclusion, we have studied hydrogen adsorption properties on Calcium-decorated, Boron-doped bilayer graphene. The empty $p_{z}$ orbital of boron is a strong electron acceptor; it can improve the adsorption energy of $\mathrm{Ca}$ to bilayer graphene. The results show that a stable and uniform structure of Ca-decorated bilayer graphene can be obtained by replacing boron doping. Our calculations show that calcium atoms can be solidly adsorbed on the interlayer $(\mathrm{Ca} / \mathrm{B} / \mathrm{Graphene})$ and outerlayer $(2 \mathrm{Ca} / \mathrm{B} / \mathrm{Graphene}$ and $3 \mathrm{Ca} / \mathrm{B} / \mathrm{Graphene})$ of $\mathrm{B}$-doped bilayer graphene. Hydrogen molecule binds with $\mathrm{Ca} / \mathrm{B} / \mathrm{Graphene}, 2 \mathrm{Ca} / \mathrm{B} / \mathrm{Graphene}$ and $3 \mathrm{Ca} / \mathrm{B} / \mathrm{Graphene}$ system with an energy that belongs to reversible usage range of $-0.2--0.6 \mathrm{eV}$. The hydrogen storage capacity of bilayer graphene was further improved due to meet the standard of best adsorption energy. Our work offers the possibility of using a metal atom modify bilayer graphene as a hydrogen storage material. 


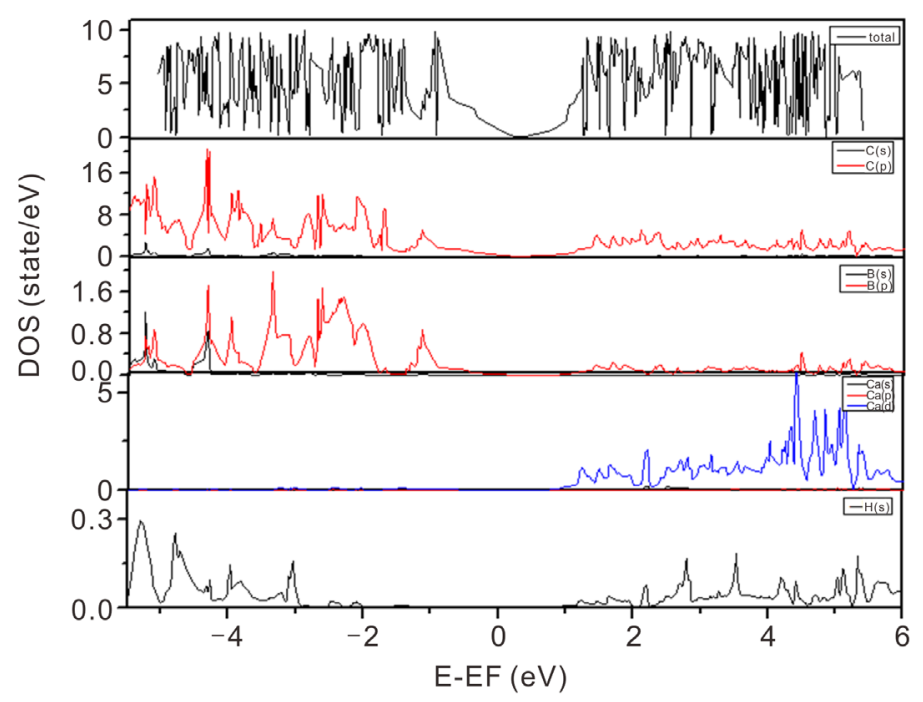

(a)

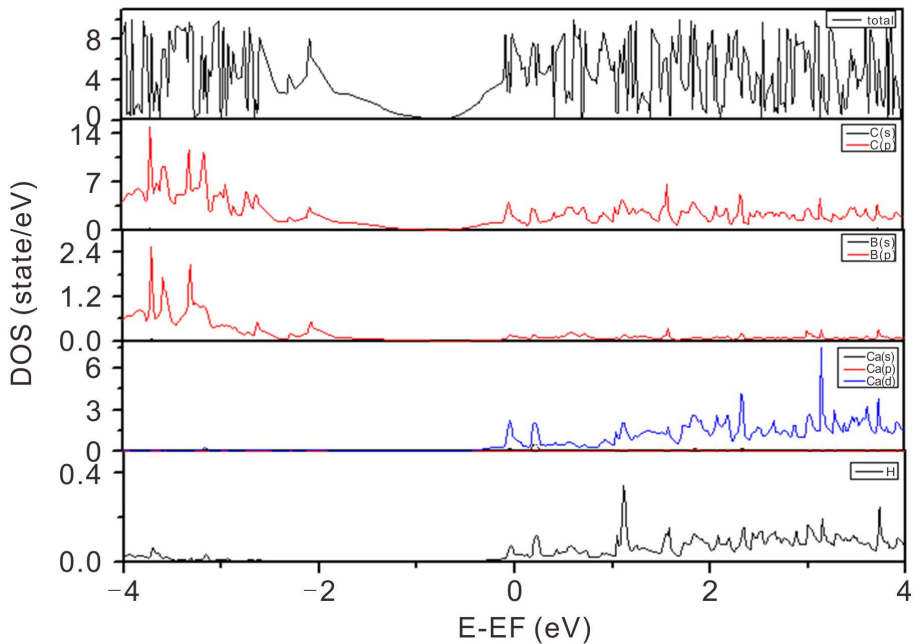

(b)

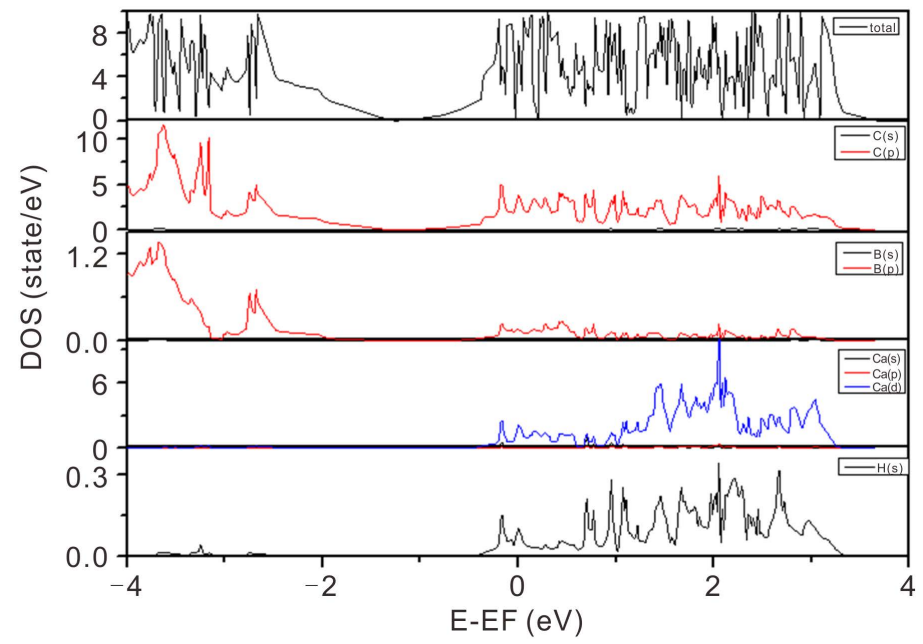

(c)

Figure 8. (a) (b) (c) shows the total and partial density of states of $1 \mathrm{H}_{2} / \mathrm{Ca} / \mathrm{B} / \mathrm{Graphene}$, $2 \mathrm{H}_{2} / 2 \mathrm{Ca} / \mathrm{B} / \mathrm{Graphene}$ and $3 \mathrm{H}_{2} / 3 \mathrm{Ca} / \mathrm{B} / \mathrm{Graphene}$, respectively. 


\section{Conflicts of Interest}

The authors declare no conflicts of interest regarding the publication of this paper.

\section{References}

[1] Schlapbach, L. and Züttel. A. (2001) Hydrogen-Storage Materials for Mobile Applications. Nature, 414, 353-358. https://doi.org/10.1038/35104634

[2] Ahluwalia, R.K., Hua, T.Q., Peng, J.K., Lasher, S., McKenney, K., Sinha, J., et al. (2010) Technical Assessment of Cryo-Compressed Hydrogen Storage Tank Systems for Automotive Applications. International Journal of Hydrogen Energy, 35, 4171-4184. https://doi.org/10.1016/j.ijhydene.2010.02.074

[3] Schoof, T., Groth, S., Vorberger, J., et al. (2015) Ab Initio Thermodynamic Results for the Degenerate Electron Gas at Finite Temperature. Physical Review Letters, 115, 130402. https://doi.org/10.1103/PhysRevLett.115.130402

[4] Sure, R. and Grimme, S. (2013) Corrected Small Basis set Hartree-Fock Method for Large Systems. Journal of Computational Chemistry, 34, 1672-1685.

https://doi.org/10.1002/jcc.23317

[5] Momen, G., Hermosilla, G., Michau, A., Pons, M., Firdaouss, M. and Hassouni, K. (2009) Hydrogen Storage in an Activated Carbon Bed: Effect of Energy Release on Storage Capacity of the Tank. International Journal of Hydrogen Energy, 34, 3799-3809. https://doi.org/10.1016/j.ijhydene.2009.03.011

[6] Bhatia, S.K. and Myers A.L. (2006) Optimum Conditions for Adsorptive Storage. Langmuir, 22, 1688-1700. https://doi.org/10.1021/la0523816

[7] Lochan, R.C. and Head-Gordon, M. (2006) Computational Studies of Molecular Hydrogen Binding Affinities: The Role of Dispersion Forces, Electrostatics, and Orbital Interactions. Physical Chemistry Chemical Physics, 8, 1357-1370. https://doi.org/10.1039/b515409j

[8] Pierson, H.O. (1993) Handbook of Carbon, Graphite, Diamond and Fullerenes. Noyes, NJ, USA.

[9] Castro, E.V., Novoselov, K.S., Morozov, S.V., et al. (2007) Biased Bilayer Graphene: Semiconductor with a Gap Tunable by the Electric Field Effect. Physical Review Letters, 99, 216802.

[10] Cai, L., Fan, J., Lin, L., et al. (2017) Influence of Donor and Acceptor Groups on the ST Energy Gap for Thermally Activated Delayed Fluorescence Emitters. Molecular Physics, 115, 809-814. https://doi.org/10.1080/00268976.2017.1287969

[11] Skone, J.H., Govoni, M. and Galli, G. (2014) Self-Consistent Hybrid Functional for Condensed Systems. Physical Review B, 89, 195112.

https://doi.org/10.1103/PhysRevB.89.195112

[12] Butler, K.T., Hendon, C.H. and Walsh, A. (2014) Electronic Chemical Potentials of Porous Metal-Organic Frameworks. Journal of the American Chemical Society, 136, 2703-2706. https://doi.org/10.1021/ja4110073

[13] Berland, K. and Hyldgaard, P. (2014) Exchange Functional that Tests the Robustness of the Plasmon Description of the van der Waals Density Functional. Physical Review B, 89, 035412. https://doi.org/10.1103/PhysRevB.89.035412

[14] Yoon, M., Yang, S., Hicke, C., Wang, E., Geohegan, D. and Zhang, Z. (2008) Calcium as the Superior Coating Metal in Functionalization of Carbon Fullerenes for High-Capacity Hydrogen Storage. Physical Review Letters, 100, 206806. 
https://doi.org/10.1103/PhysRevLett.100.206806

[15] Li, M., Li, Y.F., Zhou, Z., Shen, P.W. and Chen, Z.F. (2009) Ca-Coated Boron Fullerenes and Nanotubes as Superior Hydrogen Storage Materials. Nano Letters, 9, 1944-1948. https://doi.org/10.1021/n1900116q

[16] Lee, H., Ihm, J., Cohen, M.L. and Louie, S.G. (2009) Calcium-Decorated Carbon Nanotubes for High-Capacity Hydrogen Storage: First-Principles Calculations. Physical Review B, 80, Article ID: 115412. https://doi.org/10.1103/PhysRevB.80.115412

[17] Ataca, C., Aktürk, E. and Ciraci, S. (2009) Hydrogen Storage of Calcium Atoms Adsorbed on Graphene: First-Principles Plane Wave Calculations. Physical Review $B$, 79, Article ID: 041406. https://doi.org/10.1103/PhysRevB.79.041406

[18] Lee, H., Ihm, G., Cohen, M.L. and Louie, S.G. (2010) Calcium-Decorated Graphene-Based Nanostructures for Hydrogen Storage. Nano Letters, 10, 793-798. https://doi.org/10.1021/nl902822s

[19] Monkhorst, H.J. and Pack, J.D. (1976) Special Points for Brillouin-Zone Integrations. Physical Review B, 13, 5188-5191. https://doi.org/10.1103/PhysRevB.13.5188

[20] Yu, H.S., He, X. and Truhlar, D.G. (2016) MN15-L: A New Local Exchange-Correlation Functional for Kohn-Sham Density Functional Theory with Broad Accuracy for atoms, Molecules, and Solids. Journal of Chemical Theory and Computation, 12, 1280-1293.

[21] Omata, Y., Yamagami, Y., Tadano, K., Miyake, T. and Saito, S. (2005) Nanotube Nanoscience: A Molecular-Dynamics Study. Physica E, 29, 454-468.

https://doi.org/10.1016/j.physe.2005.06.009

[22] Fujimoto, Y. and Saito, S. (2015) Electronic Structures and Stabilities of Bilayer Graphene Doped with Boron and Nitrogen. Surface Science, 634, 57-61. https://doi.org/10.1016/j.susc.2014.11.013

[23] Dai, J., Yuan, J. and Giannozzi, P. (2009) Gas Adsorption on Graphene Doped with B, N, Al, and S: A Theoretical Study. Applied Physics Letters, 95, Article ID: 232105. https://doi.org/10.1063/1.3272008 